\title{
La hermenéutica de Eranos: una exploración de la simbólica del sentido*
}

\author{
José Luis Luna Bravo*
}

\begin{abstract}
Recibido: 14 de enero de 2015 - Revisado: 26 de febrero de 2015 -
\end{abstract}
Aprobado: 10 de abril de 2015

\section{Resumen}

El presente artículo analiza la estructura de la hermenéutica eranosiana de los símbolos, centrado en las reflexiones de Durand y Campbell, quienes retoman las tesis fundamentales de los padres espirituales de Eranos: Jung y Otto. Además cobran especial valor los estudios que Andrés Ortiz-Osés ha dedicado a Eranos. La tesis propuesta es que la hermenéutica eranosiana se basa en el carácter mediador-redundante del símbolo, de modo que es a través de la fuerza repetitiva de los simbolizantes como se precisa el sentido del simbolizado, siempre inagotable, constituyendo una simbólica redundancia coimplicante. Tras una breve reseña de la historia de Eranos, la tesis planteada se desarrolla en cuatro momentos: 1) descripción del carácter trilateral de la experiencia simbólica; 2) descripción de los tipos de signos: el símbolo como un signo lejano; 3) el carácter "infinitamente abierto" del símbolo: la redundancia como apertura; y 4) la hermenéutica eranosiana como complexio oppositorum.

Palabras clave: Eranos, símbolo, signo, hermenéutica, experiencia simbólica.

* $\quad$ Este artículo de investigación se inscribe en el marco del proyecto de investigación doctoral titulado "Phänomenologie der symbolischen Erfahrung. Der Symbolismus bei Eranos und die Phantasiewelt bei Husserl", bajo la dirección del doctor profesor Hans-Helmuth Gander, Husserl-Archiv Freiburg / Albert-Ludwigs-Universität Freiburg, Alemania, financiado por el Katholischer Akademischer Ausländer-Dienst (KAAD). DOI: http://dx.doi.org/10.15332/s0120-8454.2015.0087.08

** Doctorando en Filosofía en la Albert-Ludwigs-Universität Freiburg, Alemania, magister en Filosofía Latinoamericana y licenciado en Filosofía y Lengua Castellana por la Universidad Santo Tomás, Bogotá. Actualmente se encuentra vinculado a la Facultad de Ciencias Humanas y Sociales de la Universidad de San Buenaventura, Bogotá, y es miembro del grupo de investigación Devenir. Dirección postal: Fahnenbergplatz, 79085 Freiburg im Breisgau, Alemania, correo electrónico: joseluislunabravo@gmail.com 


\title{
The hermeneutics of Eranos: an exploration of the symbolic of the sense*
}

\author{
José Luis Luna Bravo**
}

\section{A bstract}

This article aim to analyze the structure of the Eranos' hermeneutics of symbols, focused on the comments of Durand and Campbell. They have based their comments on the fundamental thesis of the spiritual fathers of Eranos: Jung and Otto. In addition, the studies made by Andres Ortiz-Osés on the Eranos work, become particularly valuable. The thesis proposal suggests that the hermeneutic of Eranos is founded on the redundant-mediator character of the symbol. Thus, through the repetitive force of the symbols expressed as a symbolized sense, is always inexhaustible, allowing a symbolic complicant redundancy. Following the history of Eranos, the development of this proposed thesis will be performed through the following times: 1) description of the trilateral nature of the symbolic experience; 2) description of the types of signs: The symbol as a distant sign; 3). character "infinitely open" of the symbol as opening redundancy 4) hermeneutic of Eranos as complexio oppositorum.

Keywords: Eranos, symbol, sign, hermeneutic, symbolic experience.

This research paper fits into the framework of the doctoral research project entitled "Phänomenologie symbolischen der Erfahrung. Eranos bei der Symbolismus Phantasiewelt bei Husserl und die", under the direction of Professor Dr. HansHelmuth Gander, Husserl-Archiv Freiburg / Albert-Ludwigs-University Freiburg, Germany, funded by the Katholischer Akademischer Ausländer-Dienst (KAAD).

** Ph. D. student of Philosophy at the Albert-Ludwigs-University Freiburg, Germany, MA in Latin American Philosophy degree in philosophy and Spanish language by the University of St. Thomas, Bogotá. He is currently linked to the Faculty of Humanities and Social Sciences of the University of San Buenaventura, Bogotá, and is a member of the research group Becoming. Address: Fahnenbergplatz, 79085 Freiburg im Breisgau, Germany, e-mail: joseluislunabravo@gmail. com 


\title{
L'herméneutique d'Eranos: une exploration de la symbolique du sens*
}

\author{
José Luis Luna Bravoo*
}

\section{Résumé}

Cet article analyse la structure de l'herméneutique d'Eranos des symboles, centré dans les réflexions de Durand et Campbell qui reprennent les thèses fondamentales des pères spirituels d'Eranos : Jung et Otto. De plus, les recherches qu'Andrés Ortiz-Osés a réalisées sur Eranos prennent ici une dimension particulière. L'argument proposé est que l'herméneutique d'Eranos se base sur le caractère médiateur-redondant du symbole, de telle manière que c'est à travers de la force répétitive des signifiants que se précise le sens du signifié, toujours inépuisable, constituant une redondance symbolique qui co-implique. Après un bref résumé de l'histoire d'Eranos, l'argument énoncé se développe en quatre points : 1 ). description du caractère trilatéral de l'expérience symbolique; 2). description des types de signes : le symbole comme un signe lointain ; 3). le caractère "infiniment ouvert" du symbole: la redondance comme ouverture; 4 ). l'herméneutique d'Eranos comme complexio oppositorum.

Mots clés: Eranos, symbole, signe, herméneutique, expérience symbolique.

* $\quad$ Ce document de recherche inscrit dans le cadre du projet de recherche de doctorat intitulée "der Erfahrung de la Phénoménologie. Eranos bei der Symbolismus Phantasiewelt bei Husserl und die", sous la direction du professeur Dr Hans-Helmuth Gander, Husserl-Archiv Fribourg / Albert-Ludwigs-Universität Freiburg, Allemagne, financé par le Katholischer Akademischer Ausländer-Dienst (KAAD).

** Doctorat en philosophie à l'Albert-Ludwig de Fribourg-Université, l'Allemagne, la maîtrise en philosophie de l'Amérique latine diplôme en philosophie et en langue espagnole par l'Université de St. Thomas, Bogotá. Il est actuellement liée à la Faculté des Lettres et Sciences Humaines de l'Université de San Buenaventura, Bogotá, et est un membre du groupe de recherche Devenir. Adresse: Fahnenbergplatz, 79085 Freiburg im Breisgau, Allemagne, e-mail: joseluislunabravo@ gmail.com 
Analizar intelectualmente un símbolo es como pelar una cebolla para encontrarla.

Pierre Emmanuel, Considération de l'extase.

\section{Introducción: un breve mirada a la historia de Eranos}

El Círculo de Eranos, fundado en 1933 en Ascona (Suiza) por Olga FröbeKapteyn, bajo la inspiración del fenomenólogo de la religión Rudolf Otto y con la guía del psicólogo de las profundidades C.G. Jung, constituyó un espacio de encuentro de académicos de alto nivel, que ininterrumpidamente durante cincuenta y cinco años se ocuparon, en calve interdisciplinar y con una mirada universalista, de establecer un puente entre clásicos contrarios como filosofía y ciencia, mythos y logos, Oriente y Occidente, con el propósito de ofrecer una mirada holística del hombre contemporáneo a la luz de lo que el biólogo Adolf Portmann caracterizó como el centro de las investigaciones de Eranos, a saber el hombre primitivo. En 57 volúmenes, 55 anuarios y 2 ediciones conmemorativas, el Círculo de Eranos (Eranoskreis) se encargó de ofrecer una amplia visión del universo simbólico de las más antiguas culturas de la humanidad y con ello también de nuestro propio tiempo. En esta búsqueda, Andrés Ortiz-Osés distingue tres etapas en el itinerario mental de Eranos: la fundacional, de 1933 a 1946, especializada en mitología comparada; la de posguerra, de 1947 a 1971, centrada en la constitución de una antropología cultural; y la de finalización, de 1972 a 1988, con el proyecto de una hermenéutica simbólica ${ }^{1}$.

A pesar de contar la participación de pensadores como Rudolf Otto 2, C.G. Jung, Gershom Scholem, Henry Corbin, Mircea Eliade, Karl Kerényi, Paul Tillich, Hugo Rahner, Adolf Portmann, Erich Neumann, Joseph Campbell, Gilbert Durand, entre muchos otros, los encuentros de Eranos en Ascona eran más que un simple evento académico. Se trataba, como el mismo nombre Eranos lo indica, de un banquete, una comida en común en la que cada invitado demuestra ser digno de la invitación por medio de un aporte espiritual; es precisamente un punto de encuentro del que se toma parte mediante un don, un regalo. La actitud fundamental entonces que corresponde a todo Erano es la preparación

1 Para mayor información sobre la obra de Eranos véase: Ortiz-Osés, A. (2012). Hermenéutica de Eranos. Las Estructuras simbólicas del mundo. Autores, textos y temas. Hermeneusis: volumen 20. Barcelona: Anthropos; (1994, febrero) Suplementos. Materiales de trabajo intelectual. Una interpretación evaluativa de cultura. Análisis y lectura del almacén simbólico de Eranos. Barcelona: Anthropos, No. 42; (1994, febrero) El Círculo de Eranos. Una hermenéutica simbólica del sentido. Anthropos. Revista de documentación científica de la cultura, (153), Barcelona: Anthropos). En alemán se desataca: Hakl, H.T., (2001). Der verborgene Geist von Eranos. Unbekannte Begegnungen von Wissenschaft und Esoterik. Eine alternative Geistesgeschichte des 20. Jahrhunderts. Sinzheim: Scientia Nova, Verl. Neue Wiss. Más reciente, la edición de Barone, E., Riedl, M. y Tischel, A. (2004). Pioniere, Poeten, Professoren. Eranos und der Monte Verita. In der Zivilisations geschichte des 20. Jahrhunderts. Würzburg: Königshausen \& Neumann.

2 En estricto sentido Rudolf Otto no participó de los encuentros realizados a partir de 1933 en Ascona, sin embargo cumplió un rol central en la constitución del Círculo y en su posterior desarrollo, tal como lo testimonian Olga Fröbe-Kapteyn, Adolf Portamm, Gerschom Scholem, entre otros. 
del dar y simultáneamente la disposición del recibir; cada encuentro constituye pues la mediación que hace posible el ofrecimiento y la recepción del don.

La fundación del Círculo en 1933, la experiencia de la guerra y la disolución en 1988 se destacan como tres momentos fundamentales del acontecer histórico de Eranos que permiten, además de ofrecer una mirada general sobre su historia, ilustrar el mismo espíritu eranosiano.

En 1933, año del ascenso del partido nazi al poder en Alemania, se lleva a cabo el primer encuentro del Círculo de Eranos en Ascona, en el que desde el primer momento, como queda expuesto en el prólogo del primer anuario, su fundadora y primera directora Olga Fröbe-Kapteyn (1933) advierte con toda claridad el propósito a seguir:

Los encuentros de Eranos se han fijado el objetivo de la mediación entre Este y Oeste", sin embargo, aclara inmediatamente, no se trata en sí de una imitación de los métodos y teorías orientales, ni del descuido o sustitución del saber occidental sobre estas cosas, sino de que la sabiduría, simbólica y metodología oriental nos pueden ayudar al redescubrimiento de nuestros más propios valores espirituales (pp. 5-6).

Se hace patente desde el mismo inicio de los encuentros el carácter intercultural que va a marcar el desarrollo de las investigaciones, al tiempo que se deja entrever la marca específica de esta interculturalidad, a saber: no se trata de copiar o sustituir, sino de iluminar a través de la mediación.

La experiencia de la guerra va a consolidar el proyecto eranosiano, por una parte, al contar con una ininterrumpida continuidad de los encuentros, siendo de este modo testigos de la transición de una época y gestores frente a las urgentes necesidades que conllevaba tal transición. Se trata de una época que exige, en palabras de Olga Fröbe-Kapteyn (1946):

1) Una agrupación de la tradición espiritual, 2) una síntesis de todas las investigaciones científicas y 3 ) una solidaridad de toda Europa en el ámbito espiritual. Se trata entonces de 1) buscar los valores permanentes de las culturas que puedan servir de fundamento de la nueva era, 2) contar con un mirada holística del hombre y sus relaciones con el espíritu, la naturaleza y el prójimo; y 3) superar los sentimientos nacionales (p. 7).

Por otra parte, con el final de la guerra, el tema del encuentro en 1946 "Espíritu y Naturaleza" abre a Eranos al diálogo con las Ciencias Naturales y renueva su carácter universal con la presencia de investigadores procedentes de todo el mundo. De acuerdo con Olga Fröbe-Kapteyn (1949): “con ello hacemos realidad la esencia y la cualidad de la idea de lo que llamamos Eranos, pues este no es ni nacional ni continental, sino universal y humano en general" (p. 7). 
A la luz de esta nueva apertura, será el biólogo Adolf Portmann, segundo coordinador del Círculo de Eranos, en el trigésimo anuario correspondiente al año 1961, quien redefina el renovado propósito de Eranos: "con profundo respeto acoger el misterio del espíritu viviente, con buen juicio traer al lenguaje lo expresable, pero también con profundo respeto saber presente lo inexpresable, en este espíritu acontece el trabajo de Eranos" (Portmann, 1961, p. 8). Se trata entonces de una ampliación de la mediación promovida por Olga Fröbe-Kapteyn, pues no se refiere exclusivamente a Oriente y Occidente, sino a la mediación de Naturaleza y Espíritu, Expresable e Inexpresable, Racional e Irracional. Con ello se asiste a una ampliación del proyecto en el que intervienen todas las culturas bajo la idea misma de la mediación, se trata de una renovada universalidad.

Finalmente, será precisamente la restricción del proyecto de medicación cultural, ya sea en su primera concepción como mediación Oriente-Occidente bajo la dirección de Olga Fröbe-Kapteyn o en la segunda a través de la mediación Naturaleza-Espíritu bajo la batuta de Adolf Portmann, la que marcará la culminación del primer ciclo del Círculo de Eranos. Rudolf Ritsema, el último coordinador de Eranos, en el encuentro de 1988, establece como nueva ruta de las investigaciones el estudio del I Ching, Libro de las Transformaciones (Ritsema, 1988). Y tal como lo ha notado Erik Hornung (2015), "es claro que esta restricción no correspondía ni corresponde al espíritu de Eranos".

La fundación en 1933, la experiencia de la guerra e incluso la misma disolución en 1988 del Círculo de Eranos indican la impronta de mediación cultural que caracteriza tanto su obra académica, recolectada en los 57 anuarios, como su espíritu. Aquel proyecto que empezara como una mediación entre Oriente y Occidente, pasó a indagar por las relaciones entre Naturaleza y Espíritu, para finalmente disolverse al verse restringido. Con Eranos se asiste a la experiencia de la apertura y la mediación como claves de una renovada universalidad en clave intercultural.

Dicha experiencia de apertura y mediación acontece, al sentir de Eranos, en los símbolos. Y aquí llama la atención que aquello que define su labor intelectual, defina igualmente y en gran medida su planteamiento teórico, toda vez que, si se asume que el centro de su reflexión se ocupa de la experiencia simbólica en la diversidad de las tradiciones culturales, se puede afirmar que aquello que caracteriza propiamente el símbolo es su carácter mediador.

\section{El carácter trilateral de la experiencia simbólica}

Un primer acercamiento fenomenológico a la experiencia simbólica nos muestra fundamentalmente una estructura trilateral: en primer lugar nos encontramos con los simbolizantes, es decir, los símbolos materiales (objetivos) con los que tratamos en nuestra vida cotidiana. Frente a dichos símbolos objetivos materiales 
está la subjetividad constituyente de dicha experiencia, esto es, el respectivo acto simbolizante de la conciencia. Finalmente, los simbolizantes remite a un "algo otro", a lo que la subjetividad únicamente a través de ellos puede acceder. Entonces, en tercer lugar tenemos lo simbolizado. Así, la pregunta por la estructura de la experiencia simbólica comprende por lo menos los siguientes elementos: un simbolizante, un simbolizado y una conciencia simbolizante. Esta relación trilateral constituye a cada símbolo.

La tesis propuesta es grosso modo la siguiente: el acto hermenéutico en clave eranosiana se configura en la triple relación que se mantiene entre estos tres elementos, de modo que solo por medio de la dimensión de la redundancia del símbolo es posible la experiencia del sentido. Dicha redundancia acontece en los tres elementos constitutivos coimplicados de la experiencia simbólica, pues, como se verá adelante, en el redundante acto de la simbolización se requiere la aparición repetida -y siempre renovada- de los simbolizantes, en los que se encarna reiterativamente el simbolizado mismo - con una fuerza continuamente perfeccionante-.

\section{El símbolo como un signo lejano}

Como bien lo advierte Gilbert Durand, hay una gran confusión en torno a la terminología del imaginario, al punto que muchos utilizan indistintamente las palabras signo, símbolo, alegoría, parábola, mito, ícono, etc. Esto se debe, en parte, a la variedad y multidisciplinariedad propia de las investigaciones que se ocupan de los símbolos y del papel de lo imaginario en la cultura. Considerando esta situación, tratemos de aclarar, de la mano de Gilbert Durand (2007), la terminología que se refiere a la experiencia simbólica.

Según Durand, la conciencia dispone de dos modos de representarse el mundo: uno directo, en el que las cosas están presentes o pueden ser presentadas, y otro indirecto, en el que la cosa misma no puede presentarse a la sensibilidad. En este caso, el objeto es (re)presentado a la conciencia a través de una imagen. Afirma Durand (2007) que dicha diferencia no es tan tajante como parece; de ahí que:

Sería mejor decir que la conciencia dispone de distintas gradaciones de la imagen -según esta última sea una copia fiel de la sensación o simplemente indique la cosa-cuyos extremos opuestos estarían constituidos por la adecuación total, la presencia perceptiva, o bien por la inadecuación más extrema, es decir, un signo eternamente separado de su significado ${ }^{3}$ (p. 10).

3 Vale la pena advertir que en este punto tomamos distancia de lo que parece ser una suerte teoría de la imagen en torno a la idea de la percepción sensible en la propuesta de Durand. Edmund Husserl (1962) se ha ocupado con bastante detalle de mostrar las dificultades que esta postura conlleva (cf. pp. 43-44 de Ideas relativas a una fenomenología pura y una filosofía fenomenológica). 
Este signo lejano es el símbolo, de modo que el símbolo pertenece a la categoría del signo.

Los tres niveles más representativos de esta gradación de la imagen son el signo, la alegoría y el símbolo. Caracterizando estos tres niveles, podremos determinar con mayor precisión el lugar de los símbolos en la terminología general de imaginario.

Los signos remiten a un sentido que o bien está directamente presente, o bien puede ser verificado. Los signos reemplazan con economía una detallada definición conceptual y, dado que el significado es previo al significante, nada impide que este último pueda ser elegido arbitrariamente. Ahora bien, según Durand (2007), "hay casos en los que el signo debe perder su arbitrariedad teórica: cuando remite a abstracciones, en especial a cualidades espirituales o morales que es difícil presentar en "carne y hueso" (p. 11). En estos casos hablamos de alegorías. En este nivel pueden ser integrados los emblemas y las apologías. La alegoría es, entonces, una traducción concreta de una idea, que no puede ser comprendida o expresada de manera más fácil.

Por el momento, podemos distinguir dos clases de signos: 1) los signos arbitrarios, que son puramente indicativos y remiten a una realidad ausente, pero que puede ser presentada. El significado está limitado y la forma del signo es arbitraria (por lo menos teóricamente); 2) Los signos alegóricos que remiten a una realidad difícilmente representable. El significado está limitado y es traducido a través de formas menos arbitrarias.

El tercer nivel de esta gradación de la imagen corresponde a los símbolos. Se trata de signos en los que "el significado es imposible de presentar y el signo solo puede referirse a un sentido, y no a una cosa sensible" (Durand, 2007, pp. 11-12). En este caso las dos partes constitutivas del símbolo (simbolizante y simbolizado) son infinitamente abiertas. Pero, ¿qué implica este estar "infinitamente abierto"? Por un lado, que el simbolizante remite a cualidades no representables, hasta llegar a la antinomia; por ejemplo, un simbolizante como el fuego puede remitir al sentido de la salvación, la condena, la sexualidad, lo demoniaco, etc. Por otro lado, lo simbolizado puede extenderse a todo el universo concreto; por ejemplo, el sentido de lo sagrado puede estar simbolizado a través de un árbol, un animal, un planeta o una encarnación humana. El carácter infinitamente abierto del símbolo se basa en su flexibilidad. Con los signos y las alegorías tratamos con formas arbitrarias y significados limitados, mientras que en el terreno de los símbolos tenemos formas "necesarias" y sentidos inagotables.

\section{La redundancia como apertura}

En síntesis, por símbolo entendemos "un signo que remite a un significado inefable e invisible, y por eso debe encarnar concretamente esta adecuación que se le evade, y 
hacerlo mediante el juego de las redundancias míticas, rituales, iconografías, que corrigen y completan inagotablemente la inadecuación" (Durand, 2007, p. 21, cursivas del texto). Se trata entonces de un conocimiento nunca adecuado, en el que no se tiene una equivalencia indicativa entre el simbolizante y lo simbolizado.

Los simbolizantes, que pueden incluso llegar a integrar cualidades contradictorias, y lo simbolizado, que puede impregnar todo el universo, tienen en común el carácter redundante. Esta fuerza repetitiva no es tautológica sino perfeccionante, de modo que el símbolo no se corresponde con la idea de un círculo cerrado, sino más bien con una espiral, que con cada repetición precisa, focaliza su centro. Entonces, como bien lo señala C.G. Jung, mientras que la alegoría representa una idea que se distingue de sí misma, el símbolo es la idea misma, es la idea encarnada. El símbolo no va de una idea a una representación concreta, sino que el símbolo es fundamentalmente su representación, en la que aparece un sentido concreto, él es epifanía, aparición de lo inefable en y a través de los simbolizantes. El símbolo es epifanía de un misterio (Durand, 2007).

Esta epifanía se encarna a través de la fuerza redundante del símbolo. Esta fuerza redundante permite clasificar el universo simbólico en tres niveles: 1) redundancia de los gestos, es decir, los símbolos rituales; 2 ) redundancia de las relaciones lingüísticas, es decir, los mitos (en un sentido restringido); 3) redundancia de la imagen materializada, es decir, los símbolos iconográficos. Estos tres niveles de constituciones simbólicas están unidos y organizados en grandes relatos (los mitos en clave eranosiana) que ponen en escena elementos en los que se invierte una carga simbólica y de los que se exige una acción.

A modo de ilustración de la estructura de la experiencia simbólica retomemos un experimento de física analizado por Gilbert Durand (2003).

Bernard d'Espagnat muestra que cuando se emite un solo fotón (un "grano" de energía luminosa) y se pone como blanco de ese objetivo dos -jo mil!- agujeritos en una pantalla, mientras que, lógicamente, el fotón debería pasar por uno solo de esos agujeros, pasa por los dos, los mil, se difracta (...)" (pp. 52-53).

Este imagen de la física contemporánea nos permite ilustrar la distinción entre los símbolos y sus referentes, entre el vehículo y su contenido, entre simbolizantes y simbolizados; y más aún, la necesaria coimplicación -relación, junción- de los elementos constitutivos del símbolo. No hay fotón alguno que no esté traspasando los agujeros de la rejilla, como no tenemos noticia de los agujeros de no ser por el fotón luminoso emitido.

El fotón emitido corresponde al sentido, al simbolizado, mientras que los agujeritos en la pantalla ilustran a los simbolizantes. Tenemos entonces que el sentido no "pasa" por un solo agujero (sea este la ciencia, la religión, el arte, etc.), sino que por el contrario se difracta en múltiples formas simbólicas, informándonos todas ellas "algo" del simbolizado, siempre inaccesible "en sí", en tanto que 
solo tenemos noticias de él a través de los impactos en la rejilla. Estos elementos constitutivos del símbolo se coimplican. Ahora bien, no se trata de ningún modo de un tipo de noúmeno simbólico, pues el simbolizado se encarna todo en los simbolizantes, no existe fuera o más allá de ellos, aunque este se dé -y permítaseme utilizar una categoría husserliana- siempre escorzado, de ahí su inagotabilidad. En este sentido, la desaparición de una imagen, de un simbolizante, de un agujero, no implica con ello, la desaparición de su referente, pues este puede permanecer oculto en otro simbolizante, traspasando otro agujero. Igualmente la variedad y multiplicidad de formas simbólicas, simbolizantes, no significan necesariamente multiplicidad de referentes.

Más que un noúmeno en sentido kantiano, hablamos de lo numinoso en el sentido expresado por Rudolf Otto. Pues, respecto de lo simbolizado,

Únicamente puede facilitarse su comprensión de esta manera: probando a guiar al oyente por medio de sucesivas delimitaciones, hasta el punto de su propio ánimo en donde tiene que despuntar, surgir y hacérsele consciente. Este procedimiento se facilita señalando los análogos y los contrarios más característicos de lo numinoso en otras esferas del sentimiento más conocidas y familiares, y añadiendo: nuestra incógnita no es eso mismo, pero es afín a eso y opuesta a aquello. ¿No se te ofrece ahora por símisma? (Otto, 2001, p. 15).

En este sentido, el acto hermenéutico no consiste en una elucidación exhaustiva de la relación entre dos elementos conocidos, sino que se trata de aclarar la relación existente entre un término conocido (nuestros simbolizantes) y otro desconocido (simbolizado), que se coimplican incesantemente, y, en palabras de Joseph Campbell (1998), "puesto que se trata de algo esencialmente inefable, no existe metáfora o combinación de imágenes que pueda llegar a agotar todas sus implicaciones" (p. 80). La redundancia coimplicante de simbolizante y simbolizado constituye el acto de completa apertura del símbolo.

Ahora bien, si "la hermenéutica (eranosiana) es precisamente la recuperación del relacionismo simbólico propio del hombre, relacionismo simbólico que plantea la cuestión del sentido humano y no del mero significado" (Ortiz-Osés, Trías, Durand y Garagalza, 2012, p. 224), ¿cómo asumir hermenéuticamente el encuentro con los símbolos?

\section{La hermenéutica eranosiana como complexio oppositorum}

La naturaleza del símbolo nos ha mostrado un carácter bilateral entre simbolizante-simbolizado. Ahora bien, el encuentro mismo de los opuestos constituye un modo trilateral del símbolo, pues los opuestos solo lo son si mantienen la 
relación. De ahí que el motivo relevante de Eranos es la colisión de los contrarios, pero el hilo conductor de su pensamiento es la mediación de los opuestos en su complexión. De acuerdo con Ortiz-Osés et al. (2012), “en Eranos los opuestos y sus oposiciones son interpretados en sus composiciones, de modo que no son abandonados en su enemistad patente o exterior (exotérica), sino considerados en su latente amistad interior o esotérica" (p. 10).

En este sentido, la tarea de Eranos está planteada como una búsqueda incesante de la complexio oppositorum, de modo que en lugar de la bifurcación, en tanto mera división de caminos, en Eranos se yergue el concepto simbólico de encrucijada o junción de caminos, un pensamiento ambi-polivalente y no bivalente, relacional y no esquizoide. No se trata, sin embargo, de una disolución de los contrarios para arribar a una coincidencia de los opuestos (coincidentia oppositorum); se busca una resolución del símbolo no en un logos racional, sino relacional, que permita acceder a una complexión de los opuestos (complexio oppositorum), que privilegia el factor diferenciador y no confuso de los contrarios, pues "no se trata de borrar las diferencias en su indistinción coincidente, sino de amplificar nuestra identidad simple hasta llegar a su complejidad" (Ortiz-Osés et al., 2012, p. 21).

Ahora bien, dicha complejidad de los opuestos es ella misma relación, tensión, no dominadora, sino perfeccionante. Si el polo material del símbolo se deslinda de su referente que, como ya lo hemos ilustrado, es inagotable e irreductible a un determinado simbolizante, entra en una especie de inflación egológica, y se reduce a mero signo. Sin embargo, si el polo que se refiere a lo simbolizado se desliga de su carácter material (consciente) puede inundar toda la psique irracionalmente. "He aquí que el sentido emerge en la junción o juntura de inconsciente y conciencia simbólicamente. Pues en el símbolo un sentido dado literalmente en la conciencia (el significado) se reconecta a un sentido profundo, transconsciente, complejo" (Ortiz-Osés et al., 2012, p. 34).

Este símbolo de redundancia coimplicante de simbolizantes rituales, discursivos e icónicos y simbolizados inefables, invita a un encuentro que va más allá de una relación entre dos elementos conocidos -como lo es el signo y la alegoría-. Tal como lo ha notado Joseph Campbell retomando una fórmula kantiana, una hermenéutica del símbolo nos ofrece una analogía a cuatro términos: $a$ es a $b$ lo que $c$ es a $x$. En palabras de Kant (1959), no se trata de "una semejanza incompleta de dos cosas, sino una semejanza completa de dos relaciones entre cosas completamente desemejantes" (p. 58). Complementa Kant (1959) a pie de página: "por medio de tal analogía puedo, según eso, admitir un concepto de relación de las cosas que me son absolutamente desconocidas".

La hermenéutica eranosiana del símbolo no pretende entonces describir los modos en los que el elemento material del símbolo representa directamente un sentido comprensible:

Pues las analogías mitológicas, teológicas y metafísicas (las analogías simbólicas) no se refieren solo a un término parcialmente 
comprendido y comprensible, sino a la relación existente entre dos términos, uno de los cuales es empírico y el otro metafísico (y, en consecuencia, absoluta y eternamente incognoscible desde cualquier perspectiva humana) (Campbell, 1998, p. 81).

Si x es absolutamente desconocido, la naturaleza de su relación con c también es desconocida. En este sentido, la relación a - b no trata de explicar el misterio de la relación $c-x$, sino que sirve a modo de vehículo para completar la analogía. La primera relación $(a-b)$ es igualmente inagotable, pues $a^{1}$ es $a b^{1}, a^{2}$ es $a$ $b^{2}, a^{3}$ es $a b^{3}$ y así ad infinitum, lo que $c$ es a $x^{4}$. “Todas las formas de la primera relación revelan con cada vez más fuerza el ánimo de la segunda; sin embargo, conservan su inefabilidad, que más se refuerza cuanto más se revela" (Otto, 2001, p. 173). En este sentido, “analizar intelectualmente un símbolo es como pelar una cebolla para encontrarla".

"El sentido es entonces la complejificación de los simple (dado), así como la desacomplejación de los complejo (inconsciente) por cuanto concienciado" (Ortiz-Osés et al., 2012, p. 34), pues el polo material objetivo se complejiza en el acontecer del sentido simbólico, al tiempo que el sentido acontecido se descomplejiza en su materialización en y a través de los simbolizantes.

En síntesis, podemos afirmar que la estructura tríadica de la complexio oppositorum del símbolo, que viene a ser la estructura del acontecer del sentido simbólico, exige una analogía de cuatro términos para su interpretación, analogía que a su vez constituye la estructura de acto interpretativo. Partimos de una tensión de contrarios, para develar una estructura tríadica, que exige un encuentro a cuatro términos.

El símbolo es tal como El jardín de los senderos que se bifurcan de Borges, "una enorme adivinanza (cuyo tema es lo simbolizado), esa causa recóndita que le prohíbe la mención de su nombre. Omitir siempre una palabra (un sentido) [...] es quizá el modo más enfático de indicarla" (Borges, 2011, pp. 153-154).

4 A modo de ejemplo, podríamos decir: Prometeo se relaciona con los hombres; el Padre se relaciona con las creaturas; el Brahma meditador se relaciona con las visiones de su meditación; la araña se relaciona con su tela, etc..., así como Dios se relaciona con su creación. 


\section{Referencias}

Barone, E., Riedl, M. y Tischel, A. (2004). Pioniere, Poeten, Professoren. Eranos und der Monte Verita. Der Zivilisations geschichte des 20. Jahrhunderts. Würzburg: Königshausen \& Neumann.

Borges, J. L. (2011). Cuentos completos. Colombia: Lumen.

Campbell, J. (1998). El Vuelo del ganso salvaje: exploraciones en la dimensión mitológica. Barcelona: Kairós.

Durand, G. (2003). Mitos y sociedades: introducción a la mitología. Coleccion Daimon. Buenos Aires: Biblos.

Durand, G. (2007). La imaginación simbólica (2a. ed.). Biblioteca de filosofía. Buenos Aires, Madrid: Amorrortu editores.

Fröbe-Kapteyn, O. (1933). Yoga und Meditation im Osten und im Westen.(Eranos 1).

Fröbe-Kapteyn, O. (1946). Geist und Natur. (Eranos 14).

Fröbe-Kapteyn, O. (1949). Der Mensch und die mythische Welt. (Eranos 17).

Hakl, H. T. (2001). Der verborgene Geist von Eranos. Unbekannte Begegnungen von Wissenschaft und Esoterik. Eine alternative Geistesgeschichte des 20. Jahrhunderts. Sinzheim: Scientia Nova, Verl. Neue Wiss.

Husserl, E. (1962). Ideas relativas a una fenomenología pura y una filosofía fenomenológica. México: FCE.

Hornung, E. (2015, 7 de enero). Das Abenteuer Eranos. Recuperado de http:// www.eranos-ascona.ch/abenteuer_eranos.html

Kant, I. (1959). Prolegómenos. Buenos Aires: Aguilar.

Ortiz-Osés, A. Trías, E., Durand, G. y Garagalza, L. (2012). Hermenéutica de Eranos: las estructuras simbólicas del mundo. Autores, textos y temas. Hermeneusis, 20. Barcelona: Anthropos.

Ortiz-Osés, A. Trías, E., Durand, G. y Garagalza, L. (1994, febrero). Suplementos. Materiales de trabajo intelectual. Una interpretación evaluativa de cultura. Análisis y lectura del almacén simbólico de Eranos. Anthropos, (42). Barcelona: Anthropos. 
Ortiz-Osés, A. Trías, E., Durand, G. y Garagalza, L. (1994, febrero). El Círculo de Eranos. Una hermenéutica simbólica del sentido). Anthropos. Revista de Documentación Científica de la Cultura, (53). Barcelona: Anthropos.

Otto, R. (2001). Lo santo: lo racional y lo irracional en la idea de Dios. El libro de bolsillo. Humanidades. Religión y mitología, 4.106. Madrid: Editorial.

Portmann, A. (1961). Der Mensch im Spannungsfeld der Ordnungen. (Eranos 30).

Ritsema, R. (1988). Gleichklang oder Gleichzeitigkeit. (Eranos 57). 